\title{
Adaptive Optical Delay Lines for Femtosecond Laser Pulses Shaping
}

\author{
V.Ya. Molchanov ${ }^{a, *}$, S.I. Chizhikov ${ }^{a}$, O.Yu. MAKAROV ${ }^{a}$, N.P. SOlOdOVNiKOV ${ }^{a}$,

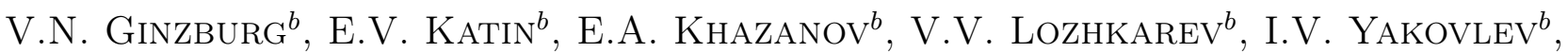 \\ I.V. EPATKO ${ }^{c}$ AND R.V. SEROV ${ }^{c}$ \\ ${ }^{a}$ Acousto-optical Research Center of Technological State University \\ "Moscow Steel and Alloys Institute" (MISA), 4, Leninsky prospect, 119049 Moscow, Russia \\ ${ }^{b}$ Institute of Applied Physics, Russian Academy of Sciences (IAP RAS) \\ 46, Ulianov str., 603950 Nizhny Novgorod, Russia \\ ${ }^{c}$ Institute of General Physics by A.M. Prokhorov, Russian Academy of Sciences (IGP RAS) \\ 38, Vavilova str., 119991 Moscow, Russia
}

\begin{abstract}
The paper is devoted to investigation of adaptive optical delay lines, intended for femtosecond pulse shaping. Experiments were performed in the sub PW laser system, based on optical parametrical chirped pulse amplification. The experimental device has revealed high spectral resolution $(0.6 \mathrm{~nm}$ at $1250 \mathrm{~nm})$ high efficiency, ( $70 \%$ over a $120 \mathrm{~nm}$ spectral width at $1250 \mathrm{~nm}$ ) and flexibility of seed pulse amplitude control. Proper shaping of the transmission function of adaptive optical delay line allows to double the femtosecond pulse spectrum band width.
\end{abstract}

PACS numbers: 42.65.Re, 42.79.Jq, 43.38.Zp

\section{Introduction}

The advancement of femtosecond laser systems would provide a wide range of new applications in physics, chemistry, biology, etc. In most of applications the quality of amplified pulse (lack of pre-pulses, high contrast ratio, pulse duration) is critical. Two femtosecond laser architectures are used now: one based on optical parametrical chirped pulse amplification (OPCPA) and the other on chirped pulse amplification. In both architectures the pulse quality is determined by a non-compensated high order dispersion and a spectral deformation in amplifiers. An adaptive device modifying spectral amplitude and phase of seed femtosecond pulse should be used to improve the duration of the compressed pulse and suppress the pre-pulses.

Acousto-optical delay lines (AODL) are almost ideal adaptive devices for independent and simultaneous control of both amplitude and phase of spectral components of femtosecond laser pulses [1-5]. An AODL operates on the principle of light diffraction by ultrasound waves in birefrigent crystal. The interaction of light with sound provides a direct incoming spectrum conversion. Collinear [6] and quasicollinear [7-10] acousto-optical interactions in crystals seem to be most suitable for pulse controlling.

\footnotetext{
* corresponding author; e-mail: v_molchanov@smtp.ru
}

The collinear acousto-optical delay lines for compression of ultrashort light pulses was first proposed by Pustovoit and Pozhar $[1,2]$ in 1986. According to their original concept the anisotropic acousto-optical interaction converts the polarization of "fast" incoming spectral component into the diffracted orthogonal "slow" one. The amplitude and the phase of each spectral component is determined by the spatial position of the sound spectral amplitudes and the frequencies in the crystal. This concept proved to be very viable. Ten years later Tournois et al. $[3,4]$ proposed the identical technical solution for femtosecond pulse shaping. Now this device (DAZZLER, Fastlite) is widely used in femtosecond laser systems.

The paratellurite single crystal is the most promising material for adaptive delay lines. The material possesses a unique combination of constants physical properties and is characterized by very high acoustical, optical and photoelastic anisotropies. In recent years the quasicollinear light-sound interaction in paratellurite became the subject matter of various articles [11-14]. However, of problems relating to an optimal delay line design remain unclarified until now. Recently an effective approach for designing delay lines was developed [5, 15]. Several experimental devices were designed and fabricated. One of these devices was preliminary tested in OPCPA laser facility.

The purpose of this paper is to perform further investigation of adaptive delay line designed according to that approach. The experiments were carried out in a 
sub PW laser system based on optical parametric pulse amplification in $\mathrm{KD}^{*} \mathrm{P}$ crystal [16].

\section{Features of adaptive optical delay line}

In the typical femtosecond OPCPA laser system the AODL is implemented between femtosecond oscillator and the stretcher. The optical parametric amplifier (OPA) is pumped by a single frequency laser operating at wavelength $\lambda_{\mathrm{p}}$. In the OPA one of the pulses diffracted by the AODF was transformed into signal radiation on the wavelength $\lambda_{\mathrm{s}}$ determined by relation $1 / \lambda_{\mathrm{s}}=1 / \lambda_{\mathrm{p}}-1 / \lambda_{i}$, where $\lambda_{i}$ - wavelength radiating by femtosecond oscillator. The adaptive control of sound wave in AODL provides the light pulse shaping both in the spectral phase delay and the amplitude.

The drawing that helps to make design of AODL [5] clear is presented in Fig. 1.

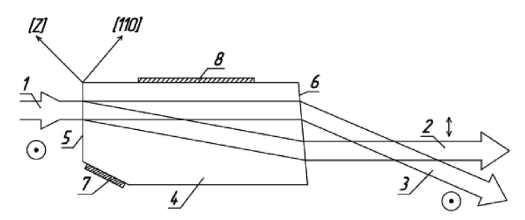

Fig. 1. Optical scheme of AODL: 1 - incoming laser radiation; 2 - diffracted beam; 3 - non-diffracted beam; 4 - paratellurite crystal; 5 - input optical facet; 6 - output facet; 7 - piezotransducer; 8 - acoustic absorber.

We assume that the input facet (5) of the crystal (4) is orthogonal to the wave vector (1) of the incoming laser radiation (Fig. 1). We consider the pure shear acoustic mode propagating in paratellurite in plane (1-10) with the polarization vector orthogonal to that plane and corresponding to very high value of merit $\mathrm{M}_{2}$. Polarization of laser radiation is shown schematically in Fig. 1.

We further assume that the acoustic wave generating by piezotransducer (7) reflects from the input facet (5) of crystal. We introduce the main parameter of AODL $\alpha_{1}$ as an angle between phase velocity of the acoustic wave reflected from the input facet of the crystal and the axis of the crystal [110]. The quasicollinear interaction takes place within the sound column reflected from the input optical facet (5) of the AODL in a such manner when phase velocity of laser radiation is strictly collinear to the group velocity of sound. After reflecting from output optical facet (6) the sound column is dissipated by acoustic absorber (8). All experimental devices were fabricated by bonding method of our own utilizing the interdiffusion in In-Au nanostructures in vacuum [17].

The angular dispersion in adaptive dispersive devices leading to an angular chirp of femtosecond laser beam originates from the angular spectral dependence of Bragg synchronism and defines the chromatic aberrations of AODL. The reduction of chromatic aberration can be done by adding a compensating prism with inverse dis- persion to the output facet (6) of the crystal as it is shown in Fig. 1.

In practice it is convenient to employ acousto-optical delay lines when the diffracted beam (2) propagates collinear to the input beam (1). In this case the angular chirp compensation will be not so perfect. The angular beam spreading will be nevertheless essentially smaller than a typical divergence of femtosecond laser beam. The tilt angle value between input (5) and onput (6) faces of the device is about 2.7 degrees.

The spectral transmission function band width of the AODL measured in a single frequency regime by optical spectrum analyzer of the Agilent Technologies at $-3 \mathrm{~dB}$ level is about $0.6 \mathrm{~nm}$ at $1550 \mathrm{~nm}$. The crystal length is $53 \mathrm{~mm}$, the optical aperture is $4 \times 4 \mathrm{~mm}, \alpha_{1}=3.8^{\circ}$. Other specifications are as follows: central laser wavelength $1250 \mathrm{~nm}$, maximum optical delay - $15 \mathrm{ps}$. The frequency of the Bragg synchronism at $1550 \mathrm{~nm}$ is $42 \mathrm{MHz}$. Owing to our own transducers bonding technology the driving RF power was extremely low (about $30 \mathrm{~mW}$ ) that permitted to control the whole femtosecond pulse spectrum up to the $200 \mathrm{~nm}$ in a multifrequency mode with efficiency exceeding $70 \%$ without temperature gradients. The general view of experimental AODL is presented in Fig. 2.

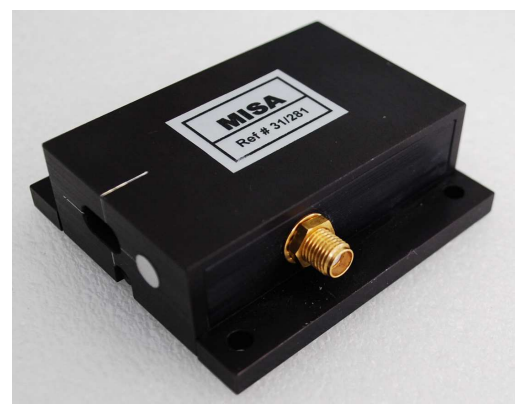

Fig. 2. General view of AODL.

\section{Pulse shaping in OPCPA laser system using AODL}

The study of an AODL operating in multifrequency regime has been carried out on the front end of a sub PW OPCPA laser system created by the IAP RAS [16]. The block diagram of the experimental system is shown in Fig. 3.

The front end of the system was founded on one double-pass OPA (4) operating at repetition rate of $1 \mathrm{~Hz}$. The femtosecond master oscillator (1) was a $\mathrm{Cr}$ :forsterite laser, generating $40 \mathrm{fs}$ pulses of $2 \mathrm{~nJ}$ at a central wavelength of $1250 \mathrm{~nm}$. An AODL cell (2) was placed between a femtosecond oscillator (1) and a stretcher (3). The diffracted beam $\mathrm{D}$ was directed toward the stretcher (3) without any deviation from the initial direction. The stretcher had the transmission band of $1000 \mathrm{~cm}^{-1}$ $(1250 \pm 60 \mathrm{~nm})$. It expanded the pulse duration up to 600 ps. The OPA (4) was pumped by a single-mode 


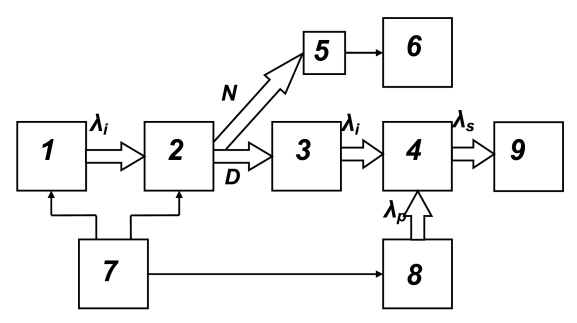

Fig. 3. Block diagram of the experimental setup: $1-$ femtosecond oscillator; 2 - AODL; 3 - stretcher; 4 OPA; 5 - photodiode; 6 - oscilloscope; 7 - synchronization system; 8 - pumping laser; 9 - spectrograph; $\mathrm{D}$ - diffracted beam; N - non-diffracted beam.

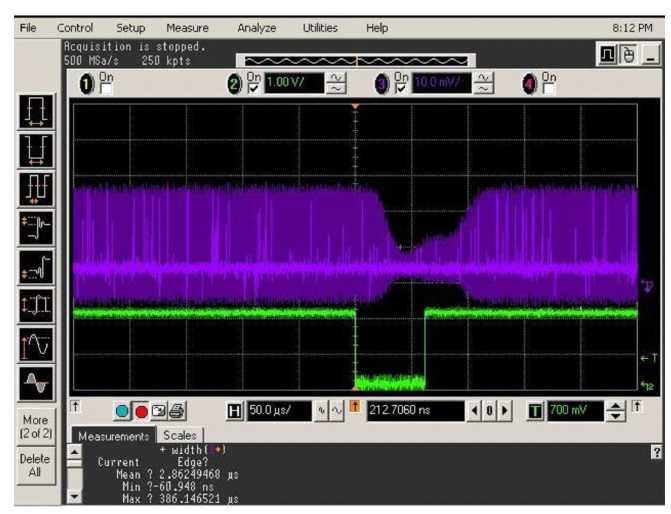

Fig. 4. Efficiency of AODL over the whole spectral band width.

single-frequency Nd:YLF laser (8) on the second harmonic at a wavelength of $\lambda_{\mathrm{p}}=527 \mathrm{~nm}$, up to $1 \mathrm{~J}$ in a 1.2 ns pulse. The nonlinear $\mathrm{KD}^{*} \mathrm{P}$ element in the OPA was $95 \mathrm{~mm}$ in length. During the first pass the OPA performed the broadband conversion of chirped pulses at $\lambda_{i}=1250 \mathrm{~nm}$ into pulses of signal radiation at $910 \mathrm{~nm}$. During the second pass the $\lambda_{\mathrm{s}}=910 \mathrm{~nm}$ radiation was amplified. The spectrum of signal radiation after the OPA was measured by a custom-made spectrograph (9).

The efficiency of AODL measurements in the multifrequency regime were performed on Infinium oscilloscope (6) of the Agilent Technologies with the use of fast photodiode (5) SIR5 of the Thorlabs.

A two-stage synchronization scheme [18] provided the simultaneous (within $\approx 50 \mathrm{ps}$ ) passage of the pulses of pumping and the pulses of single radiation through the nonlinear OPA crystals. The laser system had an optical jitter of $\pm 12 \mu$ s relative to the trigger pulse of the starting AODL.

Figure 4 shows the position of the pumping pulse within the graphic valley in the curve of non-diffracted femtosecond pulses.

The AODL was tuned to transform the whole spectrum of femtosecond pulses (the sound amplitude was constant and the sound frequency changed linearly). The time range of the valley was about $60 \mu \mathrm{s}$, which was enough

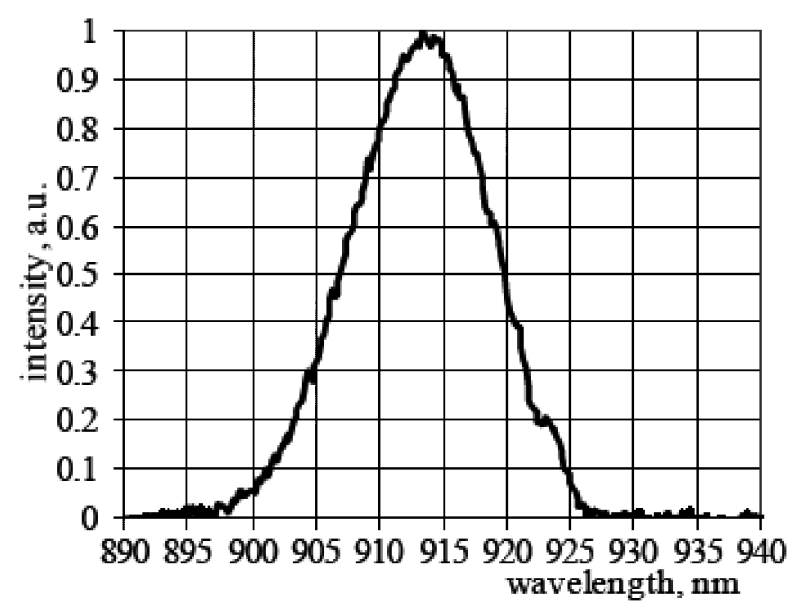

Fig. 5. The whole femtosecond pulse signal spectrum parallel processing by AODL without shaping.

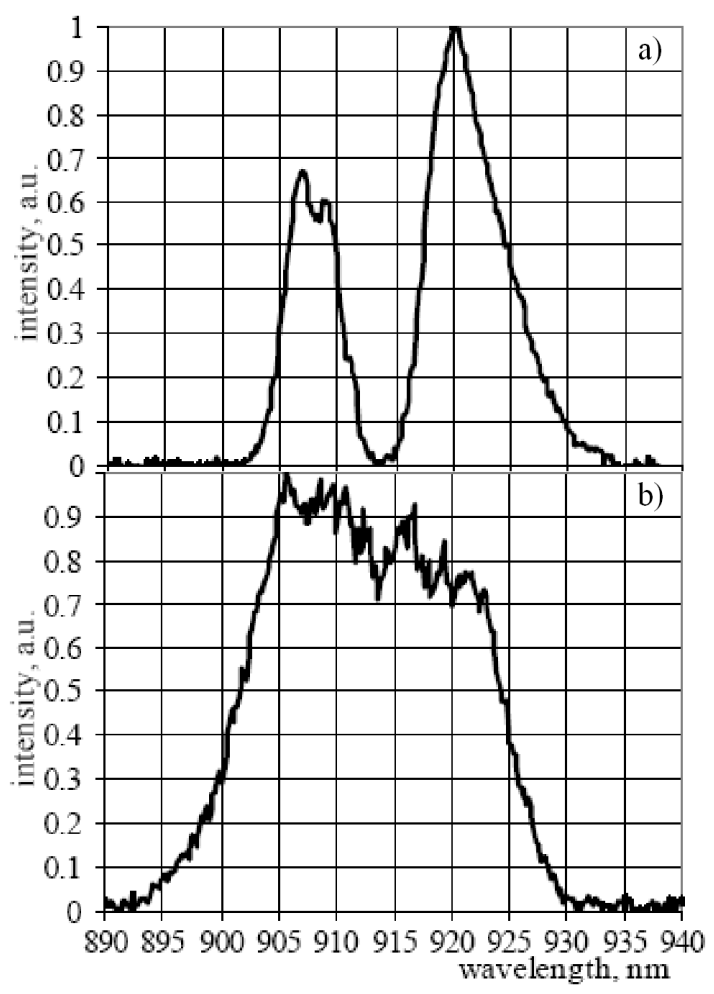

Fig. 6. Amplitude shaping of femtosecond pulse signal spectrum: (a) Gaussian hole-shaping; (b) $\pi$-shaping.

to hold all pulses injected into the OPA. The depth of valley corresponds to the AODL's efficiency of $70 \%$ over the whole spectral band.

In order to avoid the saturation of parametric amplification that may noticeably distort the pattern of spectrum of the signal the pumping pulse energy was not used above $0.4 \mathrm{~J}$.

The band width of signal spectrum without AODL was about $12 \mathrm{~nm}$ at $-3 \mathrm{~dB}$ level. This value was smaller than 
the band width of injected pulses due to temporal non-uniformity of the pumping pulse (wings of signal spectrum amplified under pumping with smaller intensity).

The spectrum of the signal in the case when the AODL was tuned to transform the whole spectrum of femtosecond pulses is presented in Fig. 5.

That spectrum is coincident to the spectrum of the signal without an AODL. Shaping of the sound amplitude distribution in form $1-d \exp \left(-\left(\lambda-\lambda_{0}\right)^{2} / w^{2}\right)$ which provides Gaussian valley $(d=1.0, w=4 \mathrm{~nm})$ produced corresponding modifications in signal laser spectrum (see Fig. 6a).

Proper selection of values of the valley's depth $(d=0.6)$ and width $(w=28 \mathrm{~nm})$ allows to provide $\pi$-shaping of the spectrum. Figure $6 \mathrm{~b}$ demonstrates clearly that the band width of signal spectrum is doubled and exceeds $23 \mathrm{~nm}$ at $-3 \mathrm{~dB}$ level.

\section{Conclusion}

Adaptive optical delay lines operating in femtosecond laser systems are the only class among all other types of acousto-optical devices providing parallel but not consistent continuous optical spectra processing.

The experimental AODL system has revealed high spectral resolution $(0.6 \mathrm{~nm}$ at $1250 \mathrm{~nm})$, high efficiency (70\% over a $120 \mathrm{~nm}$ spectral width at $1250 \mathrm{~nm}$ ), and flexibility of seed pulse amplitude control. One more important practical advantage of the designed AODL is collinearity of the diffracted beam to the incident one. The AODL application offers the prospect of optimization of the whole OPCPA laser system by increasing the efficiency of parametric amplification, expanding the spectrum band width and improving parameters of the compressed pulse.

\section{Acknowledgments}

This work is supported by the Russian Foundation for Basic Research (RFBR) under projects: No. 08-0700498, No. 08-07-12073, No. 09-07-00365 and No. 09-0700190. The authors are grateful to Yu. Kitaev from Voronegz State University for AODL driver design, as well as A. Kolesnikov from Tver State University for paratellurite crystal growth.

\section{References}

[1] V.E. Pozhar, V.I. Pustovoit, Sov. Quant. Electron. 14, 811 (1987).

[2] V.I. Pustovoit, V.E. Pozhar, Photonics Optoelectronics 2, 53 (1994).

[3] P. Tournois, Opt. Commun. 140, 245 (1997).

[4] F. Verluise, V. Laude, J.-P. Huignard, P. Tournois, Opt. Soc. Am. B 17, 138 (2000).

[5] V.Ya. Molchanov, S.I. Chizhikov, O.Yu. Makarov, N.P. Solodovnikov, V.N. Ginzburg, E.V. Katin, E.A. Khazanov, V.V. Lozhkarev, I.V. Yakovlev, Appl. Opt. 48, 1 (2009).

[6] S.E. Harris, R.W. Wallace, Opt. Soc. Am. 59, 774 (1969).

[7] J.A. Kusters, D.A. Wilson, D.L. Hammond, J. Opt. Soc. Am. 64, 434 (1974).

[8] I.-C. Chang, Electron. Lett. 28, 1255 (1992).

[9] V.B. Voloshinov, Optical Eng. 31, 2089 (1992).

[10] C.S. Qin, G.C. Huang, K.T. Chan, K.W. Cheung, Electron. Lett. 31, 1237 (1995).

[11] C.D. Tran, G.C. Huang, Opt. Eng. 38, 1143 (1999).

[12] J. Sapriel, D. Charissoux, V.B. Voloshinov, V.Ya. Molchanov, J. Lightwave Tech. 20, 892 (2002).

[13] V.V. Kludzin, A.K. Zaitsev, Opt. Commun. 219, 277 (2003).

[14] N.V. Polikarpova, V.B. Voloshinov, Proc. SPIE 5828, 25 (2004).

[15] V.Ya. Molchanov, S.I. Chizhikov, O.Yu. Makarov, in: Proc. Acoustics 08, Paris 2008, p. 827.

[16] V.V. Lozhkarev, G.I. Freidman, V.N. Ginzburg, E.V. Katin, E.A. Khazanov, A.V. Kirsanov, G.A. Luchinin, A.N. Mal'shakov, M.A. Martyanov, O.V. Palashov, A.K. Poteomkin, A.M. Sergeev, A.A. Shaykin, I.V. Yakovlev, Laser Phys. Lett. 4, 421 (2007).

[17] L.N. Magdich, V.Ya. Molchanov, Acoustooptical Devices and Their Application, Gordon and Breach, New York 1989.

[18] E.V. Katin, V.V. Lozhkarev, O.V. Palashov, E.A. Khazanov, Quantum Electron. 33, 836 (2003). 